\title{
Ethische Entscheidungsfindung im klinischen Alltag
}

Gregor Schubigera, Ruth Baumann-Hölzle ${ }^{b}$

a Prof. Dr. med,. Facharzt für Kinder- und Jugendmedizin, spez. Neonatologie, Facharzt für Intensivmedizin,

Co-Leitung Ethik-Forum Luzerner Kantonsspital

b Dr. theol., Institutsleiterin Interdisziplinäres Institut für Ethik im Gesundheitswesen der Stiftung Dialog Ethik
Korrespondenz:

Prof. Dr. med. Gregor Schubiger

Luzerner Kantonsspital

CH-6000 Luzern 16

Tel. 0414404240

gregor.schubiger[at]bluewin.ch

\section{Hintergrund}

Am Luzerner Kantonsspital (LUKS) hat sich 2011 ein interdisziplinär zusammengesetztes Ethik-Forum formiert. Diese Institution arbeitet nach dem Modell und in Kooperation mit der Stiftung Dialog Ethik. Dabei werden folgende Ziele verfolgt:

- Erarbeiten von Entscheidungsgrundlagen zu ethischen Fragen des klinischen Alltags, wie z.B. Umgang mit Patientenverfügungen und Reanimationsentscheiden, Wunsch nach assistiertem Suizid im Spital oder Spätabbrüchen von Schwangerschaften.

- Moderation von Fallbesprechungen in Teams zur strukturierten Entscheidungsfindung in schwierigen, patientenbezogenen ethischen Dilemmasituationen.

- Vermitteln von medizinethischen Grundlagen zur Reflexion neuer Herausforderungen im Gesundheitswesen und speziell in der medizinischen Entwicklung.

Als Voraussetzung für den Erwerb eines Facharzttitels sieht die Weiterbildungsordnung FMH/SIWF im Art. 163 den Erwerb von Kenntnissen im Bereich medizinische Ethik vor [1]. Die Inhalte sind im persönlichen Logbuch zu dokumentieren. Im LUKS wurde im Rahmen des Ethik-Forums ein modulares Angebot entwickelt, das

- den Facharztanwärterinnen und -anwärtern strukturierte Grundlagen zu den ethischen Prinzipen und eine möglichst fachspezifische Anwendung vermitteln soll;

- die Verantwortlichen in den verschiedenen Kliniken in der Vermittlung der geforderten Weiterbildung in Ethik unterstützt.

\section{Umsetzung}

Die Weiterbildung umfasst zwei Module und zwei auf Eigeninitiative beruhende Aktivitäten, die in einem Logbuch festgehalten und testiert werden.

Modul 1: Um möglichst vielen Ärztinnen und Ärzten die Teilnahme zu ermöglichen, werden die Grundlagen in gleicher Form 6-mal jährlich an den verschiedenen Standorten des LUKS angeboten.

Während einer Stunde sollen folgende Lernziele erreicht werden:

- Unterscheidung Sitten - Moral - Ethik kennen

- Empirisch-deskriptive und normativ-wertende Aussagen erkennen

- Ethische Dilemmas benennen und formulieren
- Organisationale Verantwortungsdimensionen einordnen

- Bedeutung des Würde- und Autonomieanspruchs einschätzen

Modul 2: Dieses wird fachspezifisch in institutionalisierte Weiterbildungsgefässe der einzelnen Kliniken eingebaut. An einem konkreten Patientenbeispiel soll ein Entscheidungsfindungsprozess aus dem entsprechenden Fachgebiet aufgearbeitet werden. Die Moderatoren verwenden dazu das 7-Schritte-Modell des Instituts Dialog Ethik [2]. Besondere Aufmerksamkeit wird dabei der Güterabwägung und der Formulierung des ethischen Dilemmas gewidmet.

Lektüre: Den Teilnehmern wird spezielle Literatur empfohlen oder zur Verfügung gestellt [z. B. 3].

Teilnahme an Fallbesprechungen: Es wird erwartet, dass Kandidatinnen und Kandidaten während der Weiterbildungszeit weitere Fallbesprechungen und Entscheidungsfindungsprozesse in ihren Teams anregen und sich aktiv in ethische Diskussionen eingeben. Zudem sollen sie unter Supervision an der Vorbereitung, Durchführung und Nachbesprechung von mindestens einem Patientengespräch mit ethischer Dimension beteiligt sein.

\section{Erfahrungen}

Im Jahr 2012 haben 80/290 (= 27\%) Assistentinnen und Assistenten das Modul 1 absolviert. Erfreulicherweise waren zusätzlich viele Kadermitglieder unter den Teilnehmern. Sieben Kliniken und drei Departemente haben in ihre Weiterbildungsveranstaltungen das Modul 2 integriert. Das Feedback war durchaus positiv. Zunehmend wird nun von Abteilungs-Teams die Möglichkeit genutzt, für schwierige Entscheidungsfindungsprozesse ethische Gespräche einzuberufen. Das Ethik-Forum stellt dazu speziell ausgebildete Moderatoren zur Verfügung.

Das Ziel besteht weiterhin darin, eine Kultur bewusster, ethischer Entscheidungsfindung innerhalb des Luzerner Kantonsspitals zu entwickeln, zu fördern und nachhaltig zu verankern.

\section{Referenzen}

1 FMH, Weiterbildungsordnung Revision 10. Januar 2013: www.fmh.ch/files/pdf8/wbo_d.pdf

2 www.dialog-ethik.ch/ueber-uns/das-modell-7-schrittedialog

3 Hope T. Medizinische Ethik. Bern: Hans Huber; 2008. 